\section{Ruptura ureteral espontánea: reporte de tres casos}

\author{
Soto-Vega E, ${ }^{1}$ Casco $S,{ }^{1}$ Andrade $D,{ }^{2}$ Macip G, ${ }^{3}$ Garay J, ${ }^{4}$ Arroyo-Kuribreña JC ${ }^{3}$
}

\section{Resumen}

ANTECEDENTES: La ruptura espontánea del uréter es poco frecuente; se asocia con dolor abdominal y debe considerarse como diagnóstico diferencial en pacientes con cólico renal, sin ninguna causa subyacente aparente. Se ha propuesto que la impactaciónretención de cálculos (litos) en la pared del uréter puede producir erosión y ulceración.

CASO CLÍNICO: Se describen tres casos de ruptura ureteral espontánea sin causa aparente. El tratamiento en dos pacientes consistió en la colocación de un catéter doble J endoscópico y en un caso se efectuó drenaje abierto para reparar la ruptura. Se prescribió tratamiento profiláctico con ceftriaxona para prevenir infecciones. Los pacientes evolucionaron adecuadamente; la urotomografía de control no mostró alteraciones ni complicaciones adicionales aparentes.

CONCLUSIONES: La ruptura ureteral es una lesión poco común; algunos reportes de ruptura espontánea sugieren como principales causas: retención urinaria, linfomas y quimioterapia.

PALABRAS CLAVE: Ruptura espontánea; uréter; dolor abdominal; cólico renal; pared ureteral; fluoroscopia.

Rev Mex Urol. 2018 Jan-Feb;78(1):34-39.

\section{Spontaneous ureteral rupture: Report of three cases}

Soto-Vega E, ${ }^{1}$ Casco $S,{ }^{1}$ Andrade $D,{ }^{2}$ Macip G, ${ }^{3}$ Garay J, ${ }^{4}$ Arroyo-Kuribreña JC ${ }^{3}$

\section{Abstract}

BACKGROUND: Spontaneous rupture of the ureter is rare. It is associated with abdominal pain and should be considered the differential diagnosis in patients with renal colic with no apparent underlying cause. Stone impaction-retention at the ureteral wall has been thought to produce erosion and ulceration.

CLINICAL CASE: Three cases of spontaneous ureteral rupture with no apparent cause were presented herein. Double-J catheter was placed in two patients and open drainage was carried out in one patient to repair the rupture. Prophylaxis with ceftriaxone was given to prevent
${ }^{1}$ Universidad Anáhuac de Puebla.

${ }^{2}$ Hospital Puebla.

${ }^{3}$ Hospital Ángeles de Puebla.

${ }^{4}$ Beneficencia Española de Puebla, Pue., México.

Recibido: enero 2017

Aceptado: diciembre 2017

Correspondencia

José Carlos Arroyo Kuribreña

jcakurologia@yahoo.com.mx

Este artículo debe citarse como

Soto-Vega E, Casco S, Andrade D, Macip G, Garay J, Arroyo-Kuribreña JC. Ruptura ureteral espontánea: reporte de tres casos. Rev Mex Urol. 2018 enefeb;78(1):34-39.

DOI: https://doi.org/10.24245/revmexurol.v78i1.1205 
infection. The patients progressed satisfactorily. Control computed tomography urography identified no alterations or apparent additional complications.

CONCLUSIONS: Ureteral rupture is a rare alteration. Urinary retention, lymphoma, and chemotherapy have been suggested as possible causes of spontaneous rupture.

KEYWORDS: Spontaneous rupture; Ureter; Abdominal pain; Renal colic; Ureter wall; Fluoroscopy.

\section{ANTECEDENTES}

La ruptura espontánea del uréter es una rara alteración, definida como la pérdida de orina no traumática, ${ }^{1}$ cuyo mecanismo fisiopatológico aún se desconoce; sin embargo, se ha propuesto que la impactación-retención de cálculos (litos) en la pared del uréter puede producir erosión y ulceración. $^{2}$

\section{CASOS CLÍNICOS}

\section{Caso 1}

Paciente masculino de 52 años de edad, quien acudió a la sala de urgencias con fuerte dolor cólico abdominal agudo, que en la escala numérica de percepción del dolor refirió 10/10. El dolor, predominantemente pélvico, en el cuadrante inferior izquierdo se irradiaba a la fosa lumbar izquierda, asociado con náuseas, diaforesis y distensión abdominal. Antecedentes médicos de importancia: diabetes mellitus en control con metformina, osteosíntesis de la columna cervical y resección transuretral de la próstata. El examen físico reveló signos vitales normales. No se encontraron signos de irritación peritoneal; el signo de Giordano izquierdo resultó positivo. La urotomografía y la fase simple no mostraron ninguna litiasis; sin embargo, en la fase de excreción se observó la extravasación de orina contrastada en el uréter proximal izquierdo (Figura 1). El paciente fue intervenido por pielografía retrógrada, con la colocación de catéter doble J. Se prescribió un esquema profiláctico de ceftriaxona ( $1 \mathrm{~g} / 12 \mathrm{~h}$ por diez días) y drenaje lumbar a cielo abierto, drenando $500 \mathrm{~mL}$ de orina clara, sin identificar ningún cálculo (lito) o cualquier causa de obstrucción adicional, ni el sitio exacto de la ruptura ureteral. El paciente fue dado de alta sin complicaciones a los 72 días de hospitalización y el catéter doble J fue retirado 4 semanas más tarde, sin referir síntomas adicionales ni complicaciones. La urotomografía de control, a los 45 días, no mostró áreas de estenosis ni secuelas, y el paciente persistió asintomático.

Una causa probable de la ruptura espontánea fue que veinticuatro horas antes de acudir a servicio médico, el paciente se encontraba en un parque de atracciones y subió varias veces a la montaña rusa, sin complicaciones inmediatas aparentes; sin embargo, existe el reporte de un caso de disección de la arteria carótida asociada con ese juego mecánico. ${ }^{3}$

\section{Caso 2}

Hombre de 58 años de edad, con historia clínica de hipertensión arterial, en tratamiento con 


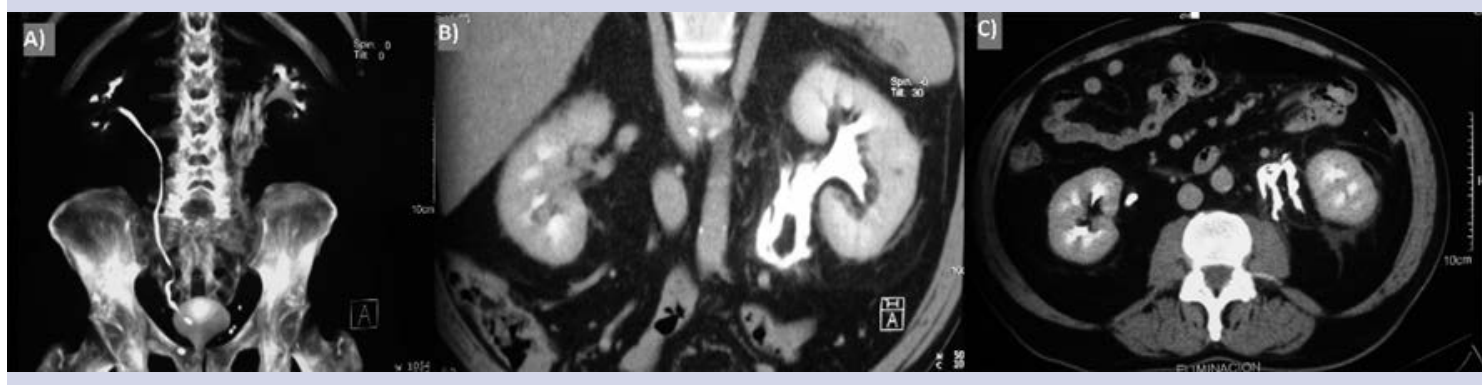

Figura 1. A) Reconstrucción ósea de la fase contrastada de la tomografía computada en la fase de eliminación, que demuestra la fuga de orina en la porción proximal del uréter. B) corte coronal de la fase de eliminación de la tomografía computada, que expone la difusión de orina contrastada en la porción proximal del uréter izquierdo. C) imagen axial de la tomografía computada en la fase de eliminación, que evidencia la fuga de orina en el uréter y se difunde a la porción posterior del riñón izquierdo.

telmisartán. Acudió al servicio de Urgencias por dolor agudo cólico en la región lumbar derecha y el flanco derecho (en la escala numérica de percepción del dolor refirió 9/10,) constante, sin náuseas, ni síntomas de la vía urinaria o irritación peritoneal. La uro-tomografía no mostró cálculos en las vías urinarias ni anormalidades; en la fase excretora se observó un escape de orina dentro de la pelvis renal derecha, sin alguna causa subyacente (Figura 2). Se realizó pielografía retrógrada y ureteroscopia flexible para confirmar la pérdida de orina en la pelvis, que mostró un eritema significativo en la cavidad renal, sin ningún sitio evidente de perforación o fuga. El paciente recibió ceftriaxona en dosis de $1 \mathrm{~g} / 12 \mathrm{~h}$ por 48 horas y cefixima en $400 \mathrm{mg} /$ día durante cinco días, además de la colocación de catéter doble J. Cuarenta y ocho horas después fue dado de alta. Su evolución fue asintomática y el catéter doble J se retiró 15 días más tarde. Luego de un mes la urotomografía de control no mostró secuelas ni áreas de estenosis.

\section{Caso 3}

Paciente masculino de 49 años de edad, con hipertensión arterial y diabetes, en tratamiento

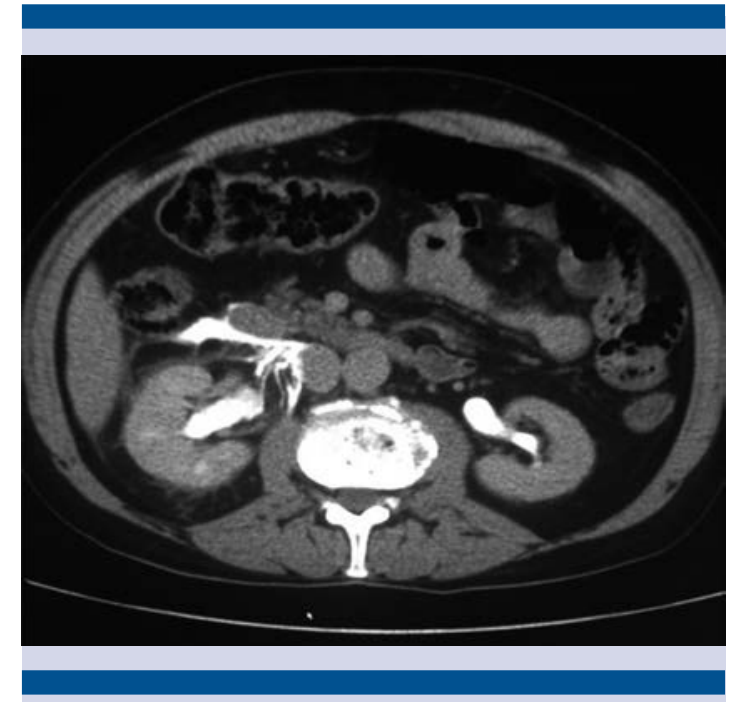

Figura 2. Tomografía axial computada, en la fase de eliminación, que demuestra la fuga de orina en la porción anterior de la pelvis renal derecha, sin litiasis comprobada.

médico. Acudió a la sala de urgencias con dolor lumbar cólico derecho repentino e intenso (escala numérica de percepción del dolor: 10/10), que se irradiaba al flanco derecho y la fosa ilíaca derecha, sin síntomas urinarios, náuseas ni fiebre. El análisis de orina mostró eritrocituria 
microscópica y en la ecografía renal se observó pielocaliectasia. La urotomografía confirmó la pielocaliectasia, además de extravasación de orina contrastada en la porción proximal del uréter derecho, sin signos de litiasis derecha, con un gran urinoma (Figura 3). El paciente se mantuvo con esquema de ceftriaxona, en dosis de $1 \mathrm{~g} / 12 \mathrm{~h}$ por 5 días. La ureteroscopia rígida no reveló litiasis ni lesión de la pared ureteral; se colocó un catéter doble J. Después de 24 h egresó sin complicaciones y tres semanas más tarde se le retiró el catéter doble J, con lo que permaneció asintomático durante su seguimiento, un mes después.

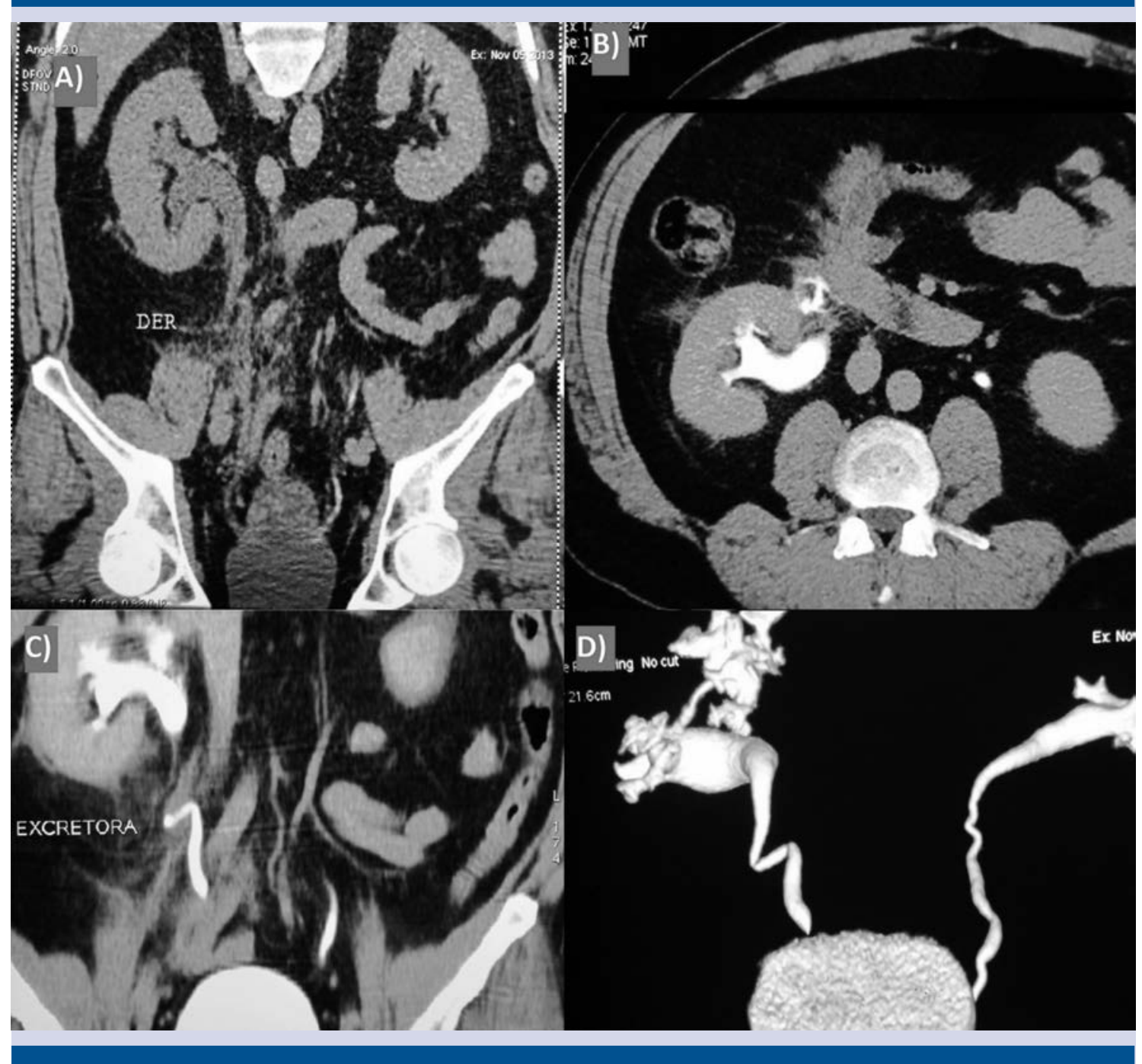

Figura 3. A) Imagen coronal de la tomografía computada en la fase simple; se observa la ausencia de urolitiasis con extravasación difusa de orina en el espacio retroperitoneal derecho, en la zona periureteral con dilatación de la pelvis renal derecha. B) Fase de eliminación, que demuestra la salida de orina contrastada dentro del hilio renal derecho, sin ninguna causa subyacente aparente. C) corte axial de la fase de eliminación de la tomografía computada, en la que se observa la dilatación de la pelvis renal derecha. D) reconstrucción de la fase de eliminación, con orina contrastada que infiltra la porción ventral de la pelvis renal derecha. 


\section{DISCUSIÓN}

La ruptura ureteral es una alteración poco común; algunos reportes de ruptura espontánea sugieren como principales causas: retención urinaria, ${ }^{4}$ linfomas y quimioterapia. ${ }^{2}$

La fisiopatología de la ruptura espontánea ureteral implica un aumento agudo de la presión intraluminal. El fondo del saco es el sitio más común de ruptura, seguido del uréter superior, cuando la presión excede de 20 a $75 \mathrm{~mm} / \mathrm{Hg}$. Otro mecanismo propuesto es la retención-impactación de cálculos (litos) en la pared ureteral, incluso su desplazamiento hacia abajo, lo que resulta en daño de la pared del uréter. $^{5}$

Entre las manifestaciones clínicas, no puede distinguirse del cólico renal asociado con náuseas, vómito, disuria, frecuencia y hematuria. ${ }^{6}$ Además, en algunos casos puede simular un abdomen agudo, con dolor repentino en esa zona y los flancos; los síntomas se relacionan con el volumen del urinoma e irritación peritoneal.

Uno de los casos expuestos experimentó síntomas vagales y signo de Giordano positivo. Ninguno de los pacientes tuvo síntomas urinarios previos. El examen físico reportó dolor abdominal y costo-vertebral en el ángulo del lado afectado. El dolor lumbar fue el síntoma principal. ${ }^{2}$ En estos casos, el diagnóstico diferencial se establece con causas de dolor abdominal, como urolitiasis, apendicitis, entre otros. ${ }^{6}$

La ruptura ureteral espontánea representa un reto diagnóstico, debido a la falta de especificidad de las manifestaciones clínicas; por lo tanto, debe considerarse como diagnóstico de exclusión. ${ }^{6}$ En nuestros pacientes no se observaron factores o registros médicos que pudieran explicar la causa subyacente de la ruptura ureteral. Como punto importante, uno de los pacientes subió previamente a la montaña rusa; sin embargo, no existe evidencia confiable que atribuya este factor como causa de la ruptura ureteral.

La urografía intravenosa y el ultrasonido representan el estudio de primera línea para la visualización de las vías urinarias; sin embargo, consideramos que la exploración tomográfica contrastada debe ser el estudio de elección, pues es más sensible y ofrece una amplia visión del sistema excretor urinario. En nuestros pacientes el diagnóstico de ruptura ureteral se estableció con la extravasación de la orina contrastada por el uréter perforado. ${ }^{7}$

En relación con el caso 3, el paciente tuvo hematuria, incluso después de realizar la ureteroscopia no se evidenció ninguna lesión o litiasis. Existen casos de ruptura ureteral asociados con carcinoma ureteral de células transicionales, en quienes la hematuria microscópica es el signo más común, por lo que debe considerarse como diagnóstico diferencial. Con la finalidad de establecer el diagnóstico, es decisiva la ureteroscopia flexible y la citología urinaria, a pesar de su baja sensibilidad y especificidad. En nuestros casos la citología urinaria no se realizó, debido a que no se encontraron anomalías uroteliales aparentes. ${ }^{8}$

Las opciones de tratamiento actual incluyen abordajes laparoscópicos y endoscópicos abiertos o radiología intervencionista: además, deben individualizarse según el estado del paciente, grado de ruptura ureteral y experiencia del cirujano. La administración profiláctica de antibióticos se recomienda en todos los pacientes para prevenir la formación de algún absceso del urinoma.

Al igual que en la bibliografía, a los pacientes de este estudio se les colocó un catéter doble J con control de contraste fluoroscópico, ya que muestra un flujo urinario sin obstrucción. Este protocolo representa el tratamiento de elección para permitir la cicatrización adecuada de la perforación ureteral y la reabsorción del uri- 
noma. ${ }^{9}$ Algunos estudios asocian este enfoque endourológico con el drenaje percutáneo. ${ }^{10}$ Las técnicas de mínima invasión, como la intervención percutánea, son la principal opción de tratamiento; sin embargo, algunos pacientes requieren drenaje abierto, como sucedió en el primer caso clínico. El uréter perforado puede causar graves complicaciones, como urinomas, estenosis ureteral, absceso retroperitoneal y sepsis. ${ }^{11}$ Como parte del tratamiento, todos los pacientes recibieron profilaxis con ceftriaxona.

\section{Conflicto de interés}

Los autores declaran no tener conflictos de interés.

\section{REFERENCIAS}

1. Chen GH, Hsiao PJ, Chang YH, et al. Spontaneous ureteral rupture and review of literature. Am J Emerg Med 2014;32(7):772-77.

2. Pampana E, Altobelli S, Morini M, Ricci A, D'Onofrio S, Simonetti G. Case Report: Spontaneous Ureteral Rupture Diagnosis and Treatment. Case Rep Radiol. 2013;2013:851-859.
3. Nouh A, Vela-Duarte D, Grobenly T, Hoganson G, Pasquale $D$, Biller J. Internal carotid artery dissection after a roller coaster ride in a 4-year-old: case report and review of the literature. Pediatr Neurol. 2015;52(3):349-351.

4. Choi S, Lee S, Kim S, et al. A rare case of upper ureter rupture: ureteral perforation caused by urinary retention. Korean J Urol 2012;53:131-133.

5. Eken A, Akbas T, Arpaci T. Spontaneous rupture of the ureter. Singapore Med J. 2015;56(2):e29-231.

6. Deng F, Liu X, Li Y, et al. Ureteral obstruction by prostate cancer leads to spontaneous ureteric rupture: a case report. Int J Clin Exp Med. 2015;8(9):16842.

7. Moak JH, Lyons MS, Lindsell CJ. Bedside renal ultrasound in the evaluation of suspected ureterolithiasis. Am J Emer Med. 2012;30(1);218-221.

8. Brimo F, Vollmer RT, Case B, et al. Accuracy of urine cytology and the significance of an atypical category. Am J Clin Pathol 2009;132:785-93.

9. Lang EK, Glorioso L. Management of urinomas by percutaneous drainage procedures. Radiol Clin North Am 1986;24( 4):551-559.

10. Akpinar $H$, Kural AR, Tüfek I, et al. Spontaneous ureteral rupture: is immediate surgical intervention always necessary? Presentation of four cases and review of the literature. J Endourol 2002;16:179-83.

11. Goldwasser B, Huszar M, Hertz M, Jonas P, Nativ O, Many M. Ureterocutaneous fistulas secondary to urinary calculous disease. Urology 1985;25:71-73.

\section{AVISO IMPORTANTE}

La Revista Mexicana de Urología se convierte en una publicación solo digital (www.revistamexicanadeurologia.org.mx) con todas las ventajas que los medios electrónicos ofrecen.

Para consultar el texto completo de los artículos deberá registrarse por una sola vez con su correo electrónico, crear una contraseña, indicar su nombre, apellidos y especialidad.

Esta información es indispensable para saber qué consulta y cuáles son sus intereses, y poder en el futuro inmediato satisfacer sus necesidades de información. 